\title{
Adverse Mandibular Bone Effects Associated with Kidney Disease Are Only Partially Corrected with Bisphosphonate and/or Calcium Treatment
}

\author{
Matthew R. Allen ${ }^{a} \quad$ Neal X. Chen ${ }^{b}$ Vincent H. Gattone II ${ }^{a}$ Sharon M. Moe ${ }^{b, c}$ \\ Departments of a Anatomy and Cell Biology and ${ }^{b}$ Medicine, Indiana University School of Medicine, and \\ 'Roudebush VA Medical Center, Indianapolis, Ind., USA
}

\section{Key Words}

Zoledronate $\cdot$ Zoledronic acid $\cdot$ C-reactive protein .

Parathyroid hormone $\cdot$ Oral bone $\cdot$ Anti-remodeling

\begin{abstract}
Background/Aims: Patients with chronic kidney disease (CKD) have a high prevalence of periodontal disease that may predispose to tooth loss and inflammation. The goal of this study was to test the hypotheses that a genetic rat model of progressive CKD would exhibit altered oral bone properties and that treatment with either bisphosphonates or calcium could attenuate these adverse changes. Methods: At 25 weeks of age, rats were treated with zoledronate (ZOL), calcium gluconate, or their combination for 5 or 10 weeks. Mandible bone properties were assessed using micro-computed tomography to determine bone volume (BV/TV) and cementum-enamel junction to alveolar crest distance (CEJAC). Results: Untreated CKD animals had significantly lower BV/TV at both $30(-5 \%)$ and $35(-14 \%)$ weeks of age and higher CEJ-AC (+27 and 29\%) compared to normal animals. CKD animals had a significantly higher parathyroid hormone (PTH) compared to normal animals, yet similar levels of Creactive protein (CRP). ZOL treatment normalized BV/TV over the first 5 weeks but this benefit was lost by 10 weeks. Calcium treatment, alone or in combination with $\mathrm{ZOL}$, was effective in normalizing BV/TV at both time points. Neither ZOL nor calcium was able to correct the higher CEJ-AC
\end{abstract}

\section{KARGER}

E-Mail karger@karger.com

www.karger.com/ajn caused by CKD. Calcium, but not ZOL, significantly reduced serum PTH, while neither treatment affected CRP. Conclusions: (i) This progressive animal model of CKD shows a clear mandibular skeletal phenotype consistent with periodontitis, (ii) the periodontitis is not associated with systemic inflammation as measured by CRP, and (iii) reducing PTH has positive effects on the mandible phenotype.

(c) 2013 S. Karger AG, Basel

\section{Introduction}

Chronic kidney disease (CKD) is often accompanied by disturbances in mineral metabolism which are classified as their own clinical entity known as CKD-mineral and bone disorder (CKD-MBD) [1]. CKD-MBD is hallmarked by altered bone remodeling and loss of bone mass throughout the skeleton, including the oral cavity $[2,3]$. Periodontal diseases, including gingivitis and periodontitis, are more prevalent in CKD populations compared to healthy individuals [4-6] and have been documented in rodent models of CKD [7]. Periodontitis is associated with alveolar bone loss, thought to be secondary to local inflammation and change in the bacterial environment. Secondary hyperparathyroidism is common in CKD, and oral bone remodeling is similar to that of cortical bone remodeling. Therefore, the etiology of periodontitis in CKD may be due to inflammation and/or secondary hy- 
Fig. 1. Experimental design: all animals began treatment at 25 weeks of age. Experiment 1 lasted 5 weeks and experiment 2 lasted 10 weeks. Animals were dosed with a single bolus of saline VEH or ZOL. Animals in calcium $(\mathrm{Ca})$ groups were fed $3 \%$ Ca gluconate in their water throughout the experimental duration.

\begin{tabular}{|c|c|c|c|c|c|c|}
\hline Experiment 1 & \multicolumn{2}{|c|}{ Normal } & \multicolumn{4}{|c|}{ CKD } \\
\hline 5-week treatment & VEH & $\begin{array}{c}\text { ZOL } \\
100 \mu \mathrm{g} / \mathrm{kg}\end{array}$ & VEH & $\begin{array}{c}\mathrm{ZOL} \\
20 \mu \mathrm{g} / \mathrm{kg}\end{array}$ & $\begin{array}{c}\text { ZOL } \\
100 \mu \mathrm{g} / \mathrm{kg}\end{array}$ & $\mathrm{Ca}$ \\
\hline Experiment 2 & \multicolumn{2}{|c|}{ Normal } & \multicolumn{4}{|c|}{ CKD } \\
\hline 10-week treatment & VEH & $\begin{array}{c}\mathrm{ZOL} \\
20 \mu \mathrm{g} / \mathrm{kg}\end{array}$ & VEH & $\begin{array}{c}\mathrm{ZOL} \\
20 \mu \mathrm{g} / \mathrm{kg}\end{array}$ & $\mathrm{Ca}$ & $\begin{array}{c}\mathrm{Ca} \\
+ \\
\mathrm{ZOL} \\
20 \mu \mathrm{kg}\end{array}$ \\
\hline
\end{tabular}

perparathyroidism [8]. If the latter is true, then therapies that lower parathyroid hormone (PTH) may also have a beneficial effect, as may other bone-sparing treatments that reduce remodeling such as bisphosphonates.

Bisphosphonates have clear efficacy in reducing bone loss in non-CKD patients [9]. International clinical practice guidelines recommend bisphosphonates use in patients with CKD stages 1-3 and normal PTH levels, but recommended not using bisphosphonates in patients with CKD stages 3-5 with biochemical evidence hyperparathyroidism [1]. The main concern for this latter population is the potential of severely suppressed bone turnover, although the limited clinical data does not universally support such an effect [10-13]. Recently, using an animal model of progressive kidney disease, we have documented that the reduction in remodeling of the tibia with zoledronic acid/zoledronate (ZOL) is similar to that of normal animals [14], although this dose failed to normalize biomechanical properties.

The effects of bisphosphonates on the oral skeleton have been extensively reviewed and discussed in recent years due to the condition of osteonecrosis of the jaw (ONJ) $[15,16]$. Although cases of ONJ have been documented in persons treated with oral bisphosphonates, the vast majority of cases have occurred in association with high-dose intravenous bisphosphonates. Indeed, oral bisphosphonates have actually been shown to benefit the oral skeleton. Oral alendronate and risedronate have each showed efficacy in attenuating periodontal-induced bone loss in the general population $[17,18]$.

The goal of this study was to characterize the oral cavity skeletal changes in this progressive kidney disease animal model. Specifically, we aimed to test the hypothesis that skeletal properties in the oral cavity would be adversely affected in animals with CKD and that bisphosphonates would attenuate these effects. We also aimed to understand the respective roles of hyperparathyroidism versus inflammation in these periodontal changes.

\section{Methods}

Animal Model and Experimental Design

A rat colony with an autosomal dominant polycystic kidney disease, maintained at the Indiana University School of Medicine, was used for this study. Male heterozygous rats $(\mathrm{Cy} /+) \mathrm{de}-$ velop characteristics of CKD (azotemia, anemia, hypertension, secondary hyperparathyroidism) around 10 weeks of age. BUN analyses were conducted in all animals at 10 weeks of age and animals with values $>40 \mathrm{mg} / \mathrm{dl}$ were considered to have CKD. These animals have a progressive rise in PTH as they age [19-21], and all animals had elevations in PTH at the time of treatment. The normal littermates of the colony were used as non-affected (normal) controls.

At 25 weeks of age (roughly a glomerular filtration rate of 25 $\mathrm{ml} / \mathrm{min}$, equivalent to human stage $4 \mathrm{CKD}$ ), animals were assigned to treatment groups within two different experiments that differed somewhat in drug dosages and also treatment duration (fig. 1).

\section{Experiment 1}

CKD animals were treated with a single dose of vehicle (VEH), ZOL (one group at 20 and another group at $100 \mu \mathrm{g} / \mathrm{kg}$ b.w.) or given $3 \%$ calcium gluconate in the drinking water. Normal animals injected with either $\mathrm{VEH}$ (saline) or a single intraperitoneal injection of ZOL (100 $\mu \mathrm{g} / \mathrm{kg}$ b.w.) served as controls. These single doses of ZOL have been shown to significantly suppress remodeling in the tibia in this model over the 5-week timeframe, and this dose of calcium significantly lowers PTH [14]. Animals were sacrificed at 30 weeks of age ( 5 weeks after treatment initiation).

\section{Experiment 2}

CKD animals were treated with a single dose of VEH, ZOL (20 $\mu \mathrm{g} / \mathrm{kg}$ b.w.), calcium gluconate, or calcium gluconate plus ZOL. Normal animals injected with either VEH (saline) or a single intraperitoneal injection of ZOL ( $20 \mu \mathrm{g} / \mathrm{kg}$ b.w.) served as controls. Animals were sacrificed at 35 weeks of age (10 weeks after treatment initiation)

In both studies, all animals were euthanized by an overdose of sodium pentobarbital. At necropsy, blood was collected by cardiac puncture. The right hemimandible was wrapped in saline-soaked gauze and frozen for imaging. All procedures were reviewed and approved by the Indiana University School of Medicine Institutional Animal Care and Use Committee. 
Computed Tomography

Morphological parameters of the mandible were assessed using high-resolution micro-CT (SkyScan 1172). Bones were wrapped in Parafilm to prevent drying during the scanning. Scans were obtained using an $\mathrm{x}$-ray source, set at $60 \mathrm{kV}$ with a $12-\mu \mathrm{m}$ pixel size. Images were reconstructed and analyzed using standard SkyScan software (NRecon and CTAn, respectively). A single slice from the central region of the first mandible molar was analyzed for total bone volume (excluding the molar and incisor) and lingual cementum-enamel to alveolar bone crest distance (CEJ-AC) as previously described $[22,23]$. This distance is roughly equivalent to the clinically assessed periodontal pocket, which is believed the nidus of inflammation (fig. 2).

Biochemical Analyses

Serum intact PTH, C-reactive protein (CRP), and TNF- $\alpha$ were measured by ELISA (Alpco, Salem, N.H., USA) according to the manufacturer's instruction. Calcium and phosphorous were measured from plasma using colorimetric methods [14].

\section{Statistics}

All analyses were run using SAS software. All data were compared using a one-way ANOVA with Fisher's LSD post hoc tests when appropriate. A p value $<0.05$ was used to determine statistical significance. Data are presented as mean and SE.

\section{Results}

Detailed long bone tissue mass and biochemical data from experiment 1 [14] and experiment 2 [24] have been previously published. In both experiments 1 and 2, there was no significant difference among groups for body mass within either experiment, while all CKD animals had significantly larger kidney masses and elevated BUN indicative of progressive kidney disease. There were no significant differences in serum calcium or phosphorous at sacrifice in experiment 1 (30 weeks) [14]. However, in experiment 2 (treated for 10 weeks), phosphorus was lower and calcium was higher in the calcium-treated CKD animals sacrificed at 35 weeks compared to other groups. At both time points, PTH was significantly higher in CKD animals $(3 \times$ higher at 30 weeks; $13 \times$ higher at 35 weeks) while CRP was not different at either time point (tables 1,2). TNF- $\alpha$ levels were undetectable in all animals. In both experiments, the calcium-treated animals had significant suppression of PTH compared to control-treated CKD animals and normal animals. Thus, the animals had progressive kidney disease and secondary hyperparathyroidism. Similar to human disease, the animals developed progressive secondary hyperparathyroidism with frank hyperphosphatemia late in the disease course. Treatment with calcium suppressed PTH and lowered phosphorus, but also increased calcium levels when the treatment was given for 10 weeks.

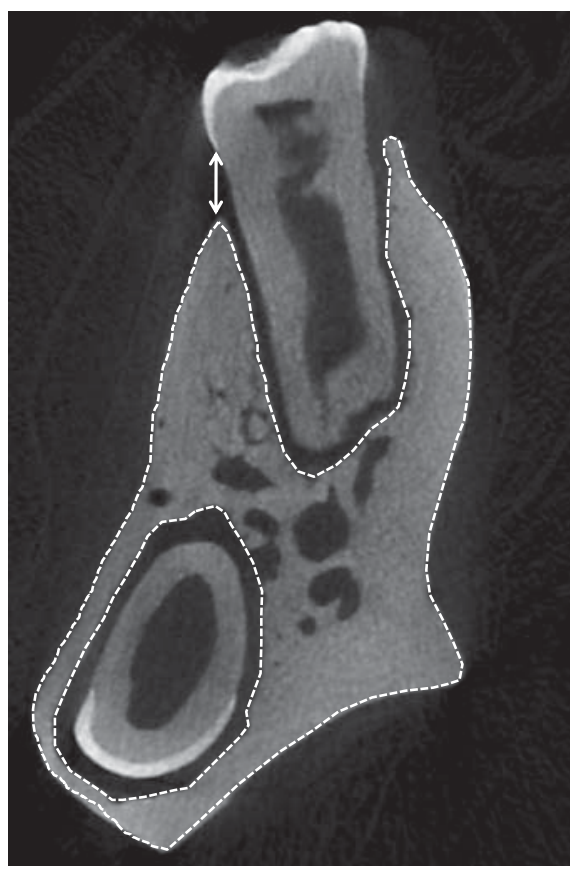

Fig. 2. CT-based morphological assessment of mandible bone. BV/ TV was calculated as the fraction of tissue that was mineralized within the entire section, excluding the dental tissue (area encompassed by the white dotted line). The CEJ-AC was calculated at the lingual surface as noted by the arrow.

Across both experiments, CKD-VEH animals displayed a clear and consistent mandibular phenotype compared to normal-VEH animals (fig. 3). Mandible bone volume/tissue volume was $6 \%$ lower and CEJ-AC distance $27 \%$ larger in the $\mathrm{CKD}$ animals compared to normal animals at 30 weeks of age. At 35 weeks of age, bone volume per tissue volume (BV/TV) was $14 \%$ lower and $\mathrm{CEJ}-\mathrm{AC} 30 \%$ higher in $\mathrm{CKD}$ animals compared to normal. ZOL did not significantly alter either periodontal assessment in normal animals of either experiment (fig. 3). ZOL treatment in experiment 1 (5 weeks of treatment) significantly improved trabecular BV/TV, at both doses with no dose response, relative to CKD-VEH. Conversely, there was no effect of $\mathrm{ZOL}$ on $\mathrm{BV} / \mathrm{TV}$ relative to $\mathrm{CKD}-\mathrm{VEH}$ in experiment 2 (10 weeks of treatment and more severe disease). ZOL was ineffective in normalizing CEJ-AC distance in both experiments. PTH levels (table 1) were significantly lower with the high dose, but not low dose. ZOL in experiment 1 was relative to CKD-VEH animals, yet was still two times higher than normal. Lowdose ZOL in experiment 2 significantly reduced PTH relative to $\mathrm{CKD}-\mathrm{VEH}$, yet it remained nearly 10 -fold higher than normal. CRP was significantly lower in ZOL-treated 
Table 1. Serum biochemistry ( 30 weeks of age -5 weeks of treatment)

\begin{tabular}{|c|c|c|c|c|c|c|c|}
\hline & \multicolumn{2}{|l|}{ Normal } & \multicolumn{4}{|l|}{ CKD } & \multirow{2}{*}{$\begin{array}{l}\text { One-way } \\
\text { ANOVA }\end{array}$} \\
\hline & VEH & ZOL $100 \mu \mathrm{g}$ & $\overline{\mathrm{VEH}}$ & ZOL $20 \mu \mathrm{g}$ & ZOL $100 \mu g$ & $\mathrm{Ca}$ & \\
\hline PTH, pg/ml & $251 \pm 34$ & $233 \pm 16$ & $853 \pm 227^{\#}$ & $593 \pm 95^{\#}$ & $558 \pm 203^{*}$ & $162 \pm 69^{*,+}$ & 0.0009 \\
\hline Range & $104-382$ & $174-303$ & $445-1,870$ & $238-950$ & $217-4,662$ & $22-420$ & \\
\hline Serum CRP, $\mu \mathrm{g} / \mathrm{ml}$ & $375 \pm 15$ & $376 \pm 15$ & $371 \pm 8$ & $353 \pm 21$ & $382 \pm 20$ & $350 \pm 9$ & 0.655 \\
\hline
\end{tabular}

Data are represented as mean $\pm \mathrm{SE}$, unless otherwise specified. $\mathrm{p}<0.05$ versus normal-VEH $\left({ }^{\#}\right), \mathrm{CKD}-\mathrm{VEH}\left({ }^{*}\right)$, and CKD-ZOL $\left({ }^{+}\right)$.

Table 2. Serum biochemistry ( 35 weeks of age -10 weeks of treatment)

\begin{tabular}{|c|c|c|c|c|c|c|c|}
\hline & \multicolumn{2}{|l|}{ Normal } & \multicolumn{4}{|l|}{ CKD } & \multirow{2}{*}{$\begin{array}{l}\text { One-way } \\
\text { ANOVA }\end{array}$} \\
\hline & VEH & ZOL $20 \mu \mathrm{g}$ & $\mathrm{VEH}$ & ZOL $20 \mu \mathrm{g}$ & $\mathrm{Ca}$ & $\mathrm{Ca}+\mathrm{ZOL}$ & \\
\hline $\mathrm{PTH}, \mathrm{pg} / \mathrm{ml}$ & $230 \pm 50 *$ & $213 \pm 17^{*}$ & $3,031 \pm 332^{\#}$ & $2,119 \pm 574^{*, \#}$ & $55 \pm 14^{*,+}$ & $69 \pm 20^{*,+}$ & $<0.0001$ \\
\hline Range & $108-613$ & $155-304$ & $972-4,760$ & $109-4,769$ & $20-4,760$ & $10-176$ & \\
\hline Serum CRP, $\mu \mathrm{g} / \mathrm{ml}$ & $459 \pm 21$ & $449 \pm 17$ & $519 \pm 26$ & $427 \pm 16^{*}$ & $528 \pm 29^{\#}$ & $525 \pm 29$ & 0.0072 \\
\hline
\end{tabular}

Data are represented as mean $\pm \mathrm{SE}$, unless otherwise specified. $\mathrm{p}<0.05$ versus normal-VEH $\left(^{\#}\right)$, CKD-VEH $(*)$, and CKD-ZOL $\left({ }^{+}\right)$.

animals of experiment 2 relative to CKD-VEH for unclear reasons.

Calcium supplementation normalized BV/TV in both experiments (fig. 3). Combining calcium with ZOL in experiment 2 produced BV/TV values comparable to calcium alone. In experiment $1, \mathrm{CEJ}-\mathrm{AC}$ was not different from normal but was also not different from CKD$\mathrm{VEH}$. In experiment 2 , neither calcium alone nor calcium combined with $\mathrm{ZOL}$ affected CEJ-AC relative to CKD-VEH. Calcium, either alone or in combination with ZOL, significantly lowered serum PTH to below normal-VEH levels. Calcium alone, in experiment 2, resulted in CRP levels that were significantly higher than normal (table 1). In the CKD animals, the PTH level was inversely correlated with the BV/TV $(\mathrm{r}=-0.77, \mathrm{p}<$ 0.001 ), although not significantly correlated with the CEJ-AC.

\section{Discussion}

Previous work describing the skeletal properties in this progressive, genetically-based CKD animal model has focused on the long bones and has documented increased bone remodeling rates, loss of bone mass, and reduced biomechanical properties $[14,21,25]$. Due to the increas- ingly prevalent recognition of dental co-morbidities associated with CKD $[5,6]$ and recent documentation of mandibular phenotype in a mouse model of CKD [7], we examined properties of the mandible that are known to be factors in the etiology of periodontal disease in our model. Our results demonstrate that this animal model that develops progressive CKD has a mandible phenotype consistent with clinical periodontal disease. Specifically, VEH-treated CKD animals had a modest but significantly lower mandible bone volume in the region of the first mandible molar compared to normal-VEH animals. CKD animals also had significantly greater CEJ-AC compared to normal animals, indicative of alveolar crest bone resorption. These skeletal differences existed in the context of significantly higher serum PTH but no difference in serum CRP, compared to normal animals. The morphological changes observed in this CKD animal model is consistent with those shown previously for both experimentally- (ligature placement) and pharmacologicallyinduced periodontal disease $[26,27]$ as well as those for another animal model of CKD [7]. Alterations in facial bones, including the mandible, have also previously been documented in a small cohort of dialysis patients where PTH is elevated [28].

Bisphosphonates as a therapy for oral bone loss have been explored and shown to be clinically effective in 

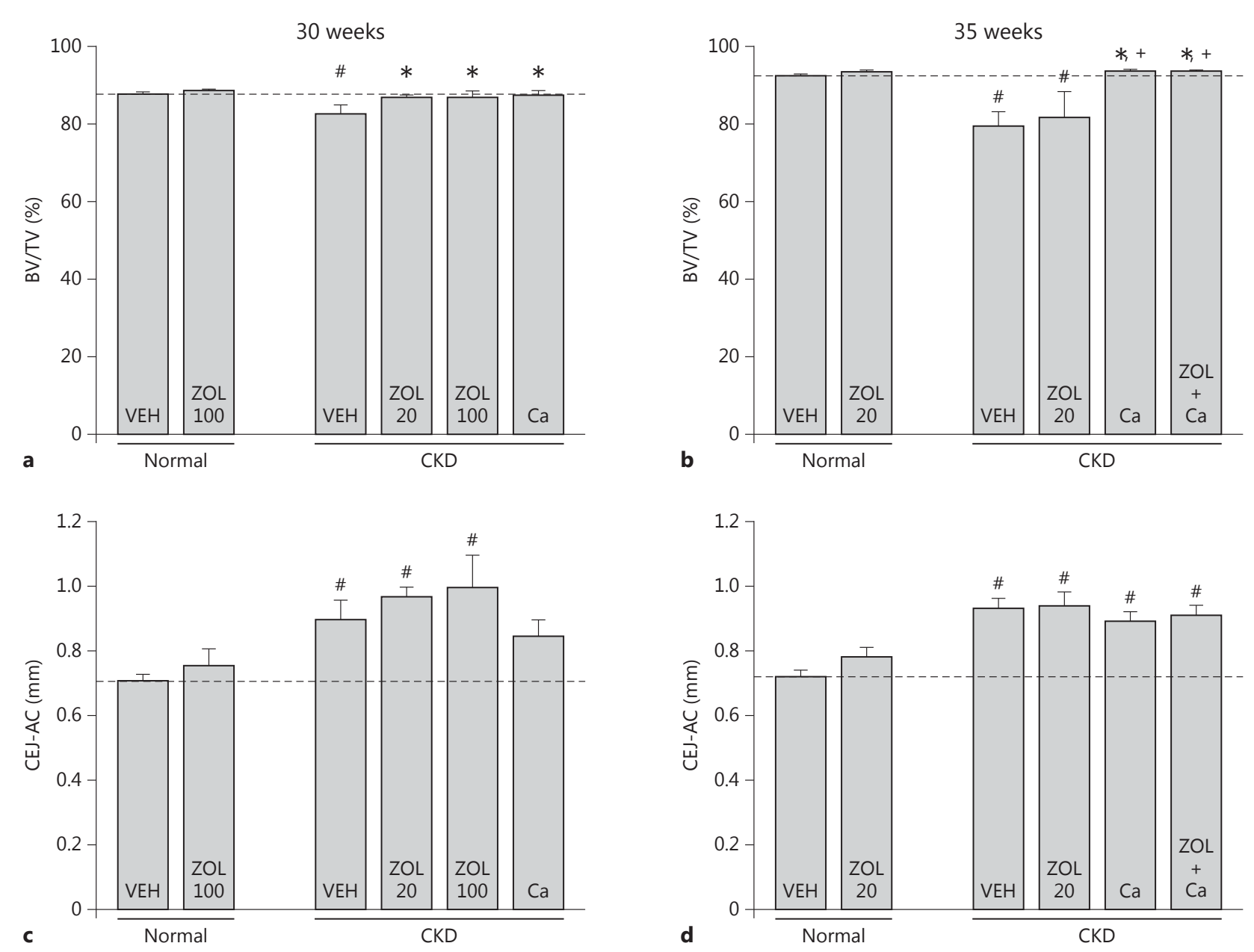

Fig. 3. Effects of $\mathrm{CKD}$ and its treatment with bisphosphonate, calcium $(\mathrm{Ca})$, or their combination on mandible trabecular bone volume $(\mathbf{a}, \mathbf{c})$ and CEJ-AC $(\mathbf{b}, \mathbf{d})$. Dotted line notes level of normal-VEH animals for reference across groups. Data are expressed as mean \pm SE. $\mathrm{p}<0.05$ versus normal-VEH $\left({ }^{\#}\right), \mathrm{CKD}-\mathrm{VEH}\left({ }^{*}\right)$, and $\left(^{+}\right) \mathrm{CKD}-\mathrm{ZOL}$.

non-CKD populations $[17,18]$. In our study, ZOL treatment, at both low and high doses, corrected the difference in mandible bone volume in the short term (5-week experiment) but not in the long term (10-week experiment). Enhanced bone volume was expected given that the mechanism of action for bisphosphonates is to reduce bone remodeling. These positive effects are consistent with the effects of ZOL at other skeletal sites in these same animals [14], as well as a wide array of other animal model conditions of bisphosphonate treatment [29]. The inability of ZOL to normalize bone volume in the 10 -week study was unexpected. Previous work has shown a single $20 \mu \mathrm{g} / \mathrm{kg}$ dose of ZOL maintained beneficial effects on tibia BV/TV bone up to 8 months post- dose in rats [30]. We interpret this as evidence that CKD alters the long-term efficacy of a single ZOL dose, likely related to the high PTH that leads to resorption of bone that is covered with the ZOL, leading to less efficacy of the drug. This could be potentially overcome by more frequent dosing - although a theoretical concern exists that with compromised renal function, increased dosing will lead to increased accumulation of the drug in the skeleton and the potential adverse effects this could manifest.

The clinical utility of bisphosphonates for treating/ preventing oral bone loss has been curtailed by ONJ, a rare but significant side effect of potent remodelingsuppressive drugs [31]. The mechanism underlying 
ONJ remains unclear and although dramatic suppression of remodeling appears to play a role, there are also a number of other co-factors that likely are involved [16]. Preclinical data show that bisphosphonates do not suppress remodeling differently in a high-turnover model of CKD compared to unaffected normal animals [14]. This would suggest that risk of ONJ, if it is related to the level of turnover suppression, would not be expected to be higher in patients with high-turnover kidney disease.

The adverse effects of CKD on the CEJ-AC were not altered with ZOL treatment. Trabecular and intracortical bone envelopes undergo bone remodeling, the coupled process of resorption followed by formation, similar to that in long bones. In these situations, inhibition of remodeling with bisphosphonates results in a small but meaningful increase in bone mass because (i) those sites that were in the process of remodeling fill in and (ii) no new remodeling sites are initiated. The cellular activity on the alveolar bone surfaces is mostly modeling, the process of resorption or formation (but not both) at a given spatial location. High PTH induced by CKD potently stimulates resorption and this is likely the mechanism underlying the greater CEJ-AC in CKD animals compared to normal $[7,28]$. Suppression of osteoclast-based modeling, as occurs with ZOL, would slow the increase of CEJ-AC distance, but would not be expected to reverse it. Non-CKD models of periodontal disease have documented that when bisphosphonate treatment is initiated before the induction of periodontal disease the CEJ-AC distance can be maintained [22]. We interpret the lack of effect in our study as evidence that the increase in CEJ-AC occurred prior to the ZOL dosing (25 weeks of age). Given that PTH levels were already elevated at this age, this is a plausible hypothesis. Modifications of CEJ-AC, a clinically-relevant parameter, would therefore necessitate either earlier treatment with an anti-resorptive or treatment with an anabolic therapy, such as PTH [32] or anti-sclerostin antibody [33].

Calcium supplementation, utilized clinically to lower phosphorus when taken with meals, and reduce elevated $\mathrm{PTH}$, provided a prolonged effect on mandible BV/TV maintenance. At both 5- and 10-week time points, animals treated with calcium had BV/TV that was comparable to normal animals, suggesting the control of PTH has a greater effect on bone preservation than the potent but acute treatment with bisphosphonate. Yet similar to ZOL, calcium supplementation was not able to restore CEJ-AC distance, again likely be- cause changes to this parameter occurred prior to the initiation of treatment.

In VEH-treated animals, serum PTH was significantly higher in CKD animals compared to normal while there was no difference in CRP, an outcome measure related to inflammation [34]. Further supporting that systemic inflammation was not an etiology in the changes were the undetectable levels TNF- $\alpha$. In the calcium-treated groups, serum PTH and bone volume were maintained; in contrast, PTH was not controlled, nor bone volume maintained in animals treated with ZOL. These observations confirm previous observations that hyperparathyroidism was associated with mandibular bone changes in a different animal model of CKD and in a small cohort of dialysis patients $[7,28]$. We also found a significant negative correlation between $\mathrm{PTH}$ and bone volume, similar to that observed in the mouse model of CKD-MBD [7]. Inflammation, globally assessed by CRP, is also associated with periodontal disease. However, inflammation may be a result rather than a cause of periodontitis. This induced systemic inflammation may be a cause of CKD, rather than CKD itself leading to periodontal disease $[8,35]$. In the present study the effects of treatment on CRP were modest and did not track with response of bone. Based on these outcomes and measurements, we conclude that PTH, not systemic inflammation, drives the bone alterations observed in this CKD model. This highlights the need to control PTH in order to reign in the oral skeletal manifestations of the disease in addition to the long bone changes.

These results should be interpreted in the context of study limitations. Our study did not include baseline controls, animals that are sacrificed at the time of treatment initiation. This would have allowed us to determine if measures such as CEJ-AC were indeed different at the start. We also did not have a group of animals that were dosed more frequently with ZOL (in experiment 2). This would have directly addressed whether controlling PTH was essential to preserving bone volume or if simply controlling osteoclasts through repeated dosing would also have been effective. We also did not have normal animals treated with calcium or calcium plus ZOL as the main reason to include the normal animals was to define the basal phenotype of the rat model.

In conclusion, we have documented that this model of progressive CKD presents a skeletal phenotype in the oral cavity consistent with clinically observed periodontal disease and that ZOL and calcium each have mixed effects as a treatment for correcting this phenotype. We also provide evidence that the bone loss of periodontal disease is related more to PTH levels than inflammation markers. 


\section{Acknowledgements}

This work was supported by NIH grant (AR058005). We would like to thank Dr. Xianming Chen, Mr. Alex Carr and Mr. Drew Brown for their assistance with the biochemical assays, breeding colony and micro-CT scanning/analysis, respectively.

\section{Disclosure Statement}

The authors have no conflicts of interest to disclose.

\section{References}

1 Moe S, Drüeke T, Cunningham J, Goodman W, Martin K, Olgaard K, et al: Definition, evaluation, and classification of renal osteodystrophy: a position statement from Kidney Disease: Improving Global Outcomes (KDIGO). Kidney Int 2006;69: 1945-1953.

-2 Malluche HH, Monier-Faugere MC: Renal osteodystrophy: what's in a name? Presentation of a clinically useful new model to interpret bone histologic findings. Clin Nephrol 2006; $65: 235-242$

-3 Barreto FC, Barreto DV, Moyses RMA, Neves CL, Jorgetti V, Draibe SA, et al: Osteoporosis in hemodialysis patients revisited by bone histomorphometry: a new insight into an old problem. Kidney Int 2006;69:1852-1857.

-4 Kshirsagar AV, Moss KL, Elter JR, Beck JD, Offenbacher S, Falk RJ: Periodontal disease is associated with renal insufficiency in the Atherosclerosis Risk In Communities (ARIC) study. Am J Kidney Dis 2005;45:650-657.

5 Proctor R, Kumar N, Stein A, Moles D, Porter S: Oral and dental aspects of chronic renal failure. J Dent Res 2005;84:199-208.

6 Brockmann W, Badr M: Kidney Disease: Chronic Kidney Disease: pharmacological considerations for the dentist. J Am Dent Assoc 2010;141:1330-1339.

7 Lee M, Chu E, El-Abbadi M, Foster B, Tompkins KA, Giachelli C, et al: Characterization of mandibular bone in a mouse model of chronic kidney disease. J Periodontol 2010;81:300309.

8 Craig RG: Interactions between chronic renal disease and periodontal disease. Oral Dis 2008; 14:1-7.

-9 Eastell R, Walsh JS, Watts NB, Siris E: Bisphosphonates for postmenopausal osteoporosis. Bone 2011;49:82-88.

-10 Jamal SA, Bauer DC, Ensrud KE, Cauley JA, Hochberg M, Ishani A, et al: Alendronate treatment in women with normal to severely impaired renal function: an analysis of the fracture intervention trial. J Bone Miner Res 2007;22:503-508.

11 Coco M: Prevention of bone loss in renal transplant recipients: a prospective, randomized trial of intravenous pamidronate. J Am Soc Nephrol 2003;14:2669-2676.

12 Jokihaara J, Pörsti IH, Kööbi P, Jolma PM, Mustonen JT, Saha HHT, et al: Treatment of experimental renal osteodystrophy with pamidronate. Kidney Int 2008;74:319-327.
13 Lomashvili KA, Monier-Faugere MC, Wang X, Malluche HH, O'Neill WC: Effect of bisphosphonates on vascular calcification and bone metabolism in experimental renal failure. Kidney Int 2009;75:617-625.

-14 Allen MR, Chen NX, Gattone VH, Chen X, Carr AJ, LeBlanc P, et al: Skeletal effects of zoledronic acid in an animal model of chronic kidney disease. Osteoporos Int 2012;24: 1471-1481.

15 Allen MR, Ruggiero SL: A review of pharmaceutical agents and oral bone health: how osteonecrosis of the jaw has affected the field; in Jensen OT (ed): Oral and Craniofacial Tissue Engineering. Hanover Park/IL, Quintessence Publishing, 2012, vol 5, pp 1-13.

16 Allen MR, Burr DB: The pathogenesis of bisphosphonate-related osteonecrosis of the jaw: so many hypotheses, so few data. J Oral Maxillofac Surg 2009;67:61-70.

17 Jeffcoat MK: Safety of oral bisphosphonates: controlled studies on alveolar bone. Int J Oral Maxillofac Implants 2006;21:349-353.

18 Lane N, Armitage GC, Loomer P, Hsieh S, Majumdar S, Wang H-Y, et al: Bisphosphonate therapy improves the outcome of conventional periodontal treatment: results of a 12-month, randomized, placebo-controlled study. J Periodontol 2005;76:1113-1122.

19 Cowley BD, Gudapaty S, Kraybill AL, Barash $\mathrm{BD}$, Harding MA, Calvet JP, et al: Autosomaldominant polycystic kidney disease in the rat. Kidney Int 1993;43:522-534.

20 Cowley BD, Grantham JJ, Muessel MJ, Kraybill $\mathrm{AL}$, Gattone VH: Modification of disease progression in rats with inherited polycystic kidney disease. Am J Kidney Dis 1996;27:865-879.

21 Moe SM, Chen NX, Seifert MF, Sinders RM, Duan D, Chen X, et al: A rat model of chronic kidney disease-mineral bone disorder. Kidney Int 2009;75:176-184.

22 Aghaloo TL, Kang B, Sung EC, Shoff M, Ronconi M, Gotcher JE, et al: Periodontal disease and bisphosphonates induce osteonecrosis of the jaws in the rat. J Bone Miner Res 2011;26: 1871-1882.

23 Kubek D, Burr D, Allen M: Ovariectomy stimulates and bisphosphonates inhibit intracortical remodeling in the mouse mandible. Orthodont Craniofac Res 2010;13:214-222.

24 Moe SM, Chen NX, Newman CL, Gattone VH II, Organ JM, Chen X, et al: A comparison of calcium to zoledronic acid for improvement of cortical bone in an animal model of CKD. J Bone Miner Res 2013 (E-pub ahead of print).
25 Moe SM, Radcliffe JS, White KE, Gattone VH, Seifert MF, Chen X, et al: The pathophysiology of early-stage chronic kidney diseasemineral bone disorder (CKD-MBD) and response to phosphate binders in the rat. J Bone Miner Res 2011;26:2672-2681.

26 Weinberg MA, Bral M: Laboratory animal models in periodontology. J Clin Periodontol 1999;26:335-340.

27 Oz H, Ebersole J: A novel murine model for chronic inflammatory alveolar bone loss. J Periodont Res 2010;45:94-99.

28 Ferrario VF, Sforza C, Dellavia C, Galassi A, Rocca Rey L, Chiarelli G, et al: Facial changes in adult uremic patients on chronic dialysis: possible role of hyperparathyroidism. Int $\mathrm{J}$ Artif Organs 2005;28:797-802.

29 Allen M, Burr D: Bisphosphonate effects on bone turnover, microdamage, and mechanical properties: what we think we know and what we know that we don't know. Bone 2011; 49:56-65.

- 30 Gasser JA, Ingold P, Venturiere A, Shen V, Green JR: Long-term protective effects of zoledronic acid on cancellous and cortical bone in the ovariectomized rat. J Bone Miner Res 2007;23:544-551.

-31 Ruggiero SL, Dodson TB, Assael LA, Landesberg R, Marx RE, Mehrotra B, et al: American Association of Oral and Maxillofacial Surgeons position paper on bisphosphonate-related osteonecrosis of the jaws - 2009 update. J Oral Maxillofac Surg 2009;67(suppl):2-12.

32 Bashutski JD, Eber RM, Kinney JS, Benavides E, Maitra S, Braun TM, et al: Teriparatide and osseous regeneration in the oral cavity. $\mathrm{N}$ Engl J Med 2010;363:2396-2405.

33 Li X, Ominsky MS, Warmington KS, Niu QT, Asuncion FJ, Barrero M, et al: Increased bone formation and bone mass induced by sclerostin antibody is not affected by pretreatment or co-treatment with alendronate in osteopenic, ovariectomized rats. Endocrinology 2011; 152:3312-3322.

34 Pepys MB, Hirschfield GM: C-reactive protein: a critical update. J Clin Invest 2003;111: 1805-1812.

35 Chambrone L, Foz AM, Guglielmetti MR, Pannuti CM, Artese HPC, Feres M, et al: Periodontitis and chronic kidney disease: a systematic review of the association of diseases and the effect of periodontal treatment on estimated glomerular filtration rate. J Clin Periodontol 2013;40:443-456. 